\title{
Renin-angiotensin system inhibitors in kidney transplantation: a benefit-risk assessment
}

\author{
Claudio Ponticelli $^{1}$ [ $\cdot$ David Cucchiari $^{1}$
}

Received: 26 September 2016 / Accepted: 2 January 2017 / Published online: 17 February 2017

(C) Italian Society of Nephrology 2017

Arterial hypertension is frequent in kidney transplant recipients and may lead to severe complications, including cardiac failure, pulmonary edema, myocardial infarction, stroke, cardiac arrhythmia, and progressive deterioration of allograft function. The pathogenesis of post-transplant hypertension is multifactorial. Apart from patients who are already hypertensive before transplantation, blood pressure can be increased by the use of calcineurin inhibitors (CNIs), corticosteroids, renal graft insufficiency, renal artery stenosis, and older age of the donor [1]. Currently, there is general agreement that the main factor responsible for post-transplant hypertension is the prolonged use of CNIs.

These drugs can induce hypertension by several mechanisms. First of all, CNIs can induce an important renal and systemic vasoconstriction by increasing the sympathetic nervous activity and the ratio between vasoconstrictive molecules (e.g., angiotensin II and endothelin) and vasodilating molecules (e.g., nitric oxide and prostacyclin). The resulting vasoconstrictive effect on afferent preglomerular arterioles decreases the glomerular filtration rate (GFR) and natriuresis leading to salt and water retention. Secondly, CNIs act directly on tubular cells by increasing sodium-chloride $(\mathrm{Na}-\mathrm{Cl})$ reabsorption, which ultimately leads to further volume expansion and arterial hypertension. This is accomplished through the overexpression of

The paper has not been published before and is not under consideration for publication anywhere else; both the authors approved the publication.

Claudio Ponticelli

ponticelli.claudio@gmail.com

1 Humanitas Clinical and Research Center, via Manzoni 56, 20089 Rozzano, MI, Italy thiazide-sensitive $\mathrm{Na}-\mathrm{Cl}$ cotransporters that is enhanced by the WNK and SPAK kinases.

In normal subjects, calcineurin can inhibit the activity of WNK and SPAK through its phosphatase activity, but the regulatory activity of calcineurin can be inhibited both by tacrolimus and cyclosporine [2, 3], so explaining the volume expansion in CNI-treated transplant recipients. Therefore, post-transplant hypertension can be considered as a sodium dependent hypertension, associated with an increase of peripheral vascular resistances.

What treatment should be used in kidney transplant recipients with hypertension? One may argue that, considering the pathogenesis of hypertension in kidney transplantation, a direct vasodilatory drug and a thiazide diuretic should represent the preferred agents to lowering blood pressure. As a matter of fact, calcium channel blockers (CCBs) are considered the agents of choice in this setting, not only because of their strong anti-hypertensive effects but also because they can reduce the rate of delayed graft function, and can improve GFR and renal allograft survival in comparison to placebo or no treatment [4]. However, the nondihydropyridine CCBs diltiazem and verapamil and the dihydropyridine nicardipine should be used with caution, since they can significantly increase the blood levels of CNI, an effect that has been exploited to reduce the dose and, consequently, the cost of CNIs, especially in low-income countries. On the other hand, $\mathrm{CCBs}$ can cause peripheral edema, and in combination with cyclosporine may worsen gingival hyperplasia, and cause constipation or gastro-esophageal reflux as a result of smooth-muscle relaxation. Since hypertension in renal transplant recipients is often difficult to control, a multi-drug regimen is often employed to reach the clinical target of $<130 / 80 \mathrm{mmHg}$. Taking into account the volume expansion that is caused by CNIs through their direct and indirect effect on renal 
tubules, a wise approach would be to add hydrochlorothiazide to a CCB-based regimen. Nevertheless, thiazides may cause volume depletion with prerenal azotemia, as well as electrolyte and acid-base disorders, and worse metabolic control in diabetic patients.

What is the role of renin-angiotensin system (RAS) inhibitors in kidney transplantation? In patients with chronic kidney disease (CKD), the use of either angiotensin-converting-enzyme (ACE)-inhibitors or angiotensin receptor blockers (ARBs) proved to slow down the progressive loss of kidney function in the long term, apart from its anti-hypertensive and cardio-protective effects. Hence, the prospect of prolonging the renal allograft survival with such a simple intervention is intriguing. However, the antihypertensive efficacy of RAS inhibitors seems to be lower in comparison to CCBs. In a randomized controlled trial comparing the CCB nifedipine with the ACE inhibitor lisinopril, nifedipine improved renal function by $20 \%$ and demonstrated better blood pressure control [5]. Moreover, in spite of the well-known beneficial effects of RAS inhibitors in CKD patients, there is currently a lack of convincing data to prove that their use can actually improve outcomes in kidney transplant recipients, and some transplant physicians are reluctant to prescribe RAS inhibitors since they can reduce hemoglobin levels and GFR. The latter effect is reversible, although it can cause problems in differentiating rejection or CNI toxicity from functional impairment in kidney transplant recipients. Cases of acute kidney injury have also been reported in kidney transplant recipients with artery stenosis or severe renal allograft dysfunction.

Some recent systematic reviews and controlled trials have pointed out the pros and cons of the use of RAS inhibitors in kidney transplantation. A systematic review of 21 randomized controlled trials in kidney transplantation reported that ACE inhibitors or ARB use, for a median period of 27 months, was associated with a significant decrease in GFR $(-5.8 \mathrm{ml} / \mathrm{min})$, hematocrit $(-3.5 \%)$ and proteinuria $(-0.47 \mathrm{gm} /$ day $)$ without significant change in the serum potassium [6]. The Kidney Disease-Improving Global Outcomes (KDIGO) guidelines reported that randomized controlled trials in kidney transplantation were not sufficiently powered statistically to determine whether ACE-inhibitors or ARB therapy can improve patient or graft survival [7]. A meta-analysis of three randomized controlled trials and two cohort studies including 20,024 kidney transplant patients showed no significant reduced risk of allograft loss or mortality among renal transplant recipients treated with RAS inhibitors [8]. In a recent multicenter, double-blind, placebo-controlled trial, 213 kidney transplant recipients with GFR $\geq 20 \mathrm{ml} / \mathrm{min}$ and proteinuria $\geq 0.2 \mathrm{~g} /$ day at 3 months after renal transplantation were randomized to either ramipril $5 \mathrm{mg}$ daily or placebo for a 4-year follow-up. The use of ramipril did not lead to a significant reduction in doubling of serum creatinine, endstage renal disease, or death. The authors concluded that the results do not support the use of ACE inhibitors with the goal of improving clinical outcomes in kidney transplant recipients with proteinuria [9]. However, the results of the study have been challenged [10]. Indeed, the final number of recruited patients was lower than expected to reach the pre-specified $80 \%$ power to detect a significant difference in primary outcome. Moreover, one-third of participants assigned to receive ramipril discontinued the drug permanently about 1 year after randomization. It has also to be noted that the low threshold of proteinuria for patient recruitment ( $>0.2 \mathrm{~g} /$ day) may have negatively affected the results, as RAS blockade has proved to be most effective in proteinuric diseases. Therefore, this study is far from being conclusive about the potential beneficial role of RAS blockade in renal transplant recipients.

In summary, although RAS inhibitors may improve arterial hypertension, proteinuria and erythrocytosis, the transplant community remains divided between the supporters and the opponents of a systematic use of RAS inhibitors in kidney transplantation. Although it is difficult to take a rigid position about their use, an early initiation to these drugs is not recommended, since it may increase the risk of delayed graft function and increase the false diagnosis of acute rejection. Whether or not to use RAS inhibitors in maintenance therapy depends on the conditions of the patient and the convictions of the caregivers. Whether or not RAS inhibitors can exert any beneficial effects on kidney allograft function, they do produce protective effects on the heart and are therefore indicated in patients with heart failure or ischemic heart disease. However, caution should be used with the so-called "dual-blockade" (ACE inhibitor plus $\mathrm{ARB}$, or $\mathrm{ACE}$ inhibitor plus aliskiren) since it does not add beneficial effects and can even increase the risk of side effects. We suggest not to use RAS inhibitors in patients with an estimated GFR $\leq 40 \mathrm{ml} / \mathrm{min}$, since they can increase the risk of hyperkalemia and can worsen anemia and serum creatinine levels. We recommend the use of a single ACE inhibitor or ARB in patients with proteinuria, while we are against the use of dual block in renal transplant patients. At any rate, when the decision of using these agents is taken, a duplex Doppler is mandatory prior to prescribing the drug in order to detect a possible renal artery stenosis. Moreover, careful monitoring of serum creatinine, potassium and hemoglobin levels is recommended during treatment with RAS inhibitors. In the future, the nephrology community should strive to develop better and riskprofile designed randomized controlled trials to investigate the role of RAS blockade in kidney transplant recipients, as the available data are not conclusive. As the pathogenesis of hypertension in kidney transplantation is multifactorial and still remains to be defined completely, the selection of 
a different profile of patients, with a higher level of proteinuria and a higher renin plasma activity, may prove to be the successful approach to definitively demonstrate the utility of RAS inhibition in slowing renal function loss in kidney transplant recipients.

\section{Compliance with ethical standards}

Conflict of interest No potential conflict of interest was reported by the authors.

Ethical approval This article does not contain any studies with human participants performed by any of the authors.

\section{References}

1. Ponticelli C, Cucchiari D, Graziani G (2011) Hypertension in kidney transplant recipients. Transpl Int 24:523-533

2. Hoorn EJ, Walsh SB, McCormick JA et al (2011) The calcineurin inhibitor tacrolimus activates the renal sodium chloride cotransporter to cause hypertension. Nat Med 17:1304-1309

3. Hoorn EJ, Walsh SB, McCormick JA et al (2012) Pathogenesis of calcineurin inhibitor-induced hypertension. J Nephrol 25:269-275
4. Cross NB, Webster AC, Masson P (2009) Antihypertensives for kidney transplant recipients: systematic review and meta-analysis of randomized controlled trials. Transplantation 88:7-18

5. Midtvedt K, Hartmann A, Foss A et al (2001) Sustained improvement of renal graft function for two years in hypertensive renal transplant recipients treated with nifedipine as compared to lisinopril. Transplantation 72:1787-1792

6. Hiremath S, Fergusson D, Doucette S et al (2007) Renin angiotensin system blockade in kidney transplantation: a systematic review of the evidence. Am J Transplant 7:2350-2360

7. Kidney Disease: Improving Global Outcomes (KDIGO) Transplant Work Group (2009) KDIGO clinical practice guideline for the care of kidney transplant recipients. Am J Transplant 9(Suppl 3):S1-155

8. Cheungpasitporn W, Thongprayoon C, Mao MA (2016) The effect of renin-angiotensin system inhibitors on kidney allograft survival: a systematic review and meta-analysis. N Am J Med Sci 8:291-296

9. Knoll GA, Fergusson D, Chasse M et al (2016) Ramipril versus placebo in kidney transplant patients with proteinuria: a multicentre, double-blind, randomised controlled trial. Lancet Diabetes Endocrinol 4:318-326

10. Toto RD (2016) The role of RAAS inhibitors in kidney transplantation. Nat Rev Nephrol 12:129-131 